\title{
Tao Li, Mitsunori Ogihara, George Tzanetakis (eds.). Music data mining
}

\author{
Chapman \& Hall/CRC, 2012, xxiv+360. US \$ 89.95, GB £ 57.99, \\ $€$ 73.99, ISBN: 978-1-4398-3552-4
}

\section{Ricardo Maronna}

Published online: 6 June 2012

(C) Springer-Verlag 2012

Table of contents:

Part I. Fundamental Topics: 1. Music data mining: an introduction (Tao Li, LeiLi), 2. Audio feature extraction (George Tzanetakis).

Part II. Classification: 3. Auditory sparse coding (Steven R. Ness, Thomas C. Walters, Richard F. Lyon), 4. Instrument recognition (Jayme Garcia Arnal Barbedo), 5. Mood and emotional classification (Mitsunori Ogihara, Youngmoo Kim), 6. Zipf's law, power laws, and music aesthetics (Bill Manaris, Patrick Roos, Dwight Krehbiel,Thomas Zalonis, J.R. Armstrong).

Part III. Social Aspects of Music Data Mining: 7. Web- and community-based music information extraction (Markus Schedl), 8. Indexing music with tags (Douglas Turnbull), 9. Human computation for music classification (Edith Law).

Part IV. Advanced Topics: 10. Hit song science (François Pachet), 11. Symbolic data mining in musicology (IanKnopke, Frauke Jürgensen).

This book contains a collection of papers written by some of the leading researchers in the field of music information retrieval (MIR). The first two chapters are a general introduction to the area. The other nine are to a great extent self-contained. Each introduces a specific subject and then surveys the different approaches to the subject, with abundant bibliographical references.

The names "support vector machines", "k-nearest neighbors" and "clustering" appear many times, but the concrete examples of their application are to be found in the references. The book can be considered as a useful survey for the reader specifically interested in MIR.

\footnotetext{
R. Maronna ( $\varangle)$

University of La Plata, La Plata, Argentina

e-mail:rmaronna@retina.ar
} 